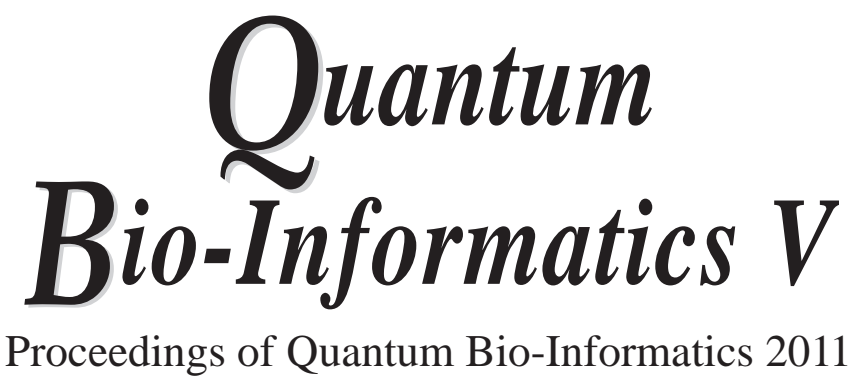




\section{QP-PQ: Quantum Probability and White Noise Analysis*}

Managing Editor: W. Freudenberg Advisory Board Members: L. Accardi, T. Hida, R. Hudson and

K. R. Parthasarathy

\section{QP-PQ: Quantum Probability and White Noise Analysis}

Vol. 30: Quantum Bio-Informatics V eds. L. Accardi, W. Freudenberg and M. Ohya

Vol. 29: Quantum Probability and Related Topics eds. L. Accardi and F. Fagnola

Vol. 28: Quantum Bio-Informatics IV From Quantum Information to Bio-Informatics eds. L. Accardi, W. Freudenberg and M. Ohya

Vol. 27: Quantum Probability and Related Topics eds. R. Rebolledo and M. Orszag

Vol. 26: Quantum Bio-Informatics III From Quantum Information to Bio-Informatics eds. L. Accardi, W. Freudenberg and M. Ohya

Vol. 25: Quantum Probability and Infinite Dimensional Analysis Proceedings of the 29th Conference eds. H. Ouerdiane and A. Barhoumi

Vol. 24: Quantum Bio-Informatics II From Quantum Information to Bio-informatics eds. L. Accardi, W. Freudenberg and M. Ohya

Vol. 23: Quantum Probability and Related Topics eds. J. C. García, R. Quezada and S. B. Sontz

Vol. 22: Infinite Dimensional Stochastic Analysis eds. A. N. Sengupta and P. Sundar

Vol. 21: Quantum Bio-Informatics From Quantum Information to Bio-Informatics eds. L. Accardi, W. Freudenberg and M. Ohya

Vol. 20: Quantum Probability and Infinite Dimensional Analysis eds. L. Accardi, W. Freudenberg and M. Schürmann

Vol. 19: Quantum Information and Computing eds. L. Accardi, M. Ohya and N. Watanabe

Vol. 18: Quantum Probability and Infinite-Dimensional Analysis From Foundations to Applications eds. M. Schürmann and U. Franz 


\section{$Q P-P Q$ \\ Quantum Probability and White Noise Analysis \\ Volume XXX}

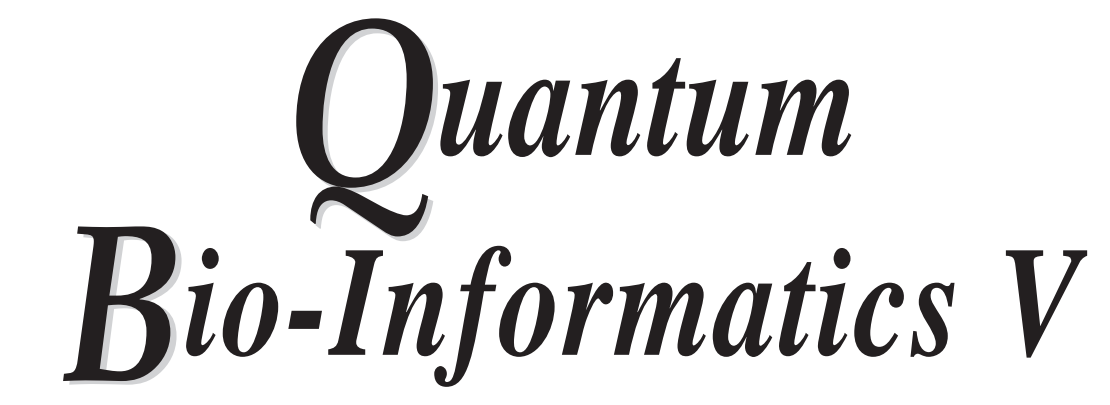

Proceedings of Quantum Bio-Informatics 2011

Tokyo University of Science, Japan $\quad 7-12$ March 2011

Editors

\section{Luigi Accardi}

Università di Roma "Tor Vergata", Italy

\section{Wolfgang Freudenberg}

Brandenburgische Technische Universität Cottbus, Germany

\section{Masanori Ohya}

Tokyo University of Science, Japan 
Published by

World Scientific Publishing Co. Pte. Ltd.

5 Toh Tuck Link, Singapore 596224

USA office: 27 Warren Street, Suite 401-402, Hackensack, NJ 07601

UK office: 57 Shelton Street, Covent Garden, London WC2H 9HE

\section{British Library Cataloguing-in-Publication Data}

A catalogue record for this book is available from the British Library.

QP-PQ: Quantum Probability and White Noise Analysis - Vol. 30

QUANTUM BIO-INFORMATICS V

Proceedings of the Quantum Bio-Informatics 2011

Copyright $@ 2013$ by World Scientific Publishing Co. Pte. Ltd.

All rights reserved. This book, or parts thereof, may not be reproduced in any form or by any means, electronic or mechanical, including photocopying, recording or any information storage and retrieval system now known or to be invented, without written permission from the Publisher.

For photocopying of material in this volume, please pay a copying fee through the Copyright Clearance Center, Inc., 222 Rosewood Drive, Danvers, MA 01923, USA. In this case permission to photocopy is not required from the publisher.

ISBN 978-981-4460-01-9

Printed in Singapore by Mainland Press Pte Ltd. 


\section{PREFACE}

This volume is based on the fifth international conference of quantum bio-informatics held at the QBI Center of Tokyo University of Sciences.

The purpose of the conference is towards new stage making interdisciplinary bridges in mathematics, physics, information and life sciences, in particular, research for new paradigm for information science and life science on the basis of quantum theory.

More than 100 researchers in various fields such as mathematics, physics, information and biology come from all over the world. The conference was held for nearly one week, and we had a lot of fruitful discussion. In this fifth conference, particular attention is come up on quantum entanglement, simulation of bio-systems, brain function, quantum like dynamics and adaptive systems. Most of speakers gave care to the relation between their own topics and the mystery of life.

The papers submitted in this volume are all refereed, whose contents are related to one of the following subjects:

(1) Mathematics of Cryptography and its related topics

(2) Quantum algorithm and computation

(3) Quantum entanglement

(4) Quantum entropy and information dynamics

(5) Quantum dynamics and time operator

(6) Stochastic dynamics and white noise analysis

(7) Brain activity

(8) Quantum like models and PD game

(9) Quantum physics and superconductivity

(10) Quantum tomography and sufficiency

(11) Adaptation in Plants

(12) Alignment of sequences

Luigi Accardi

Wolfgang Freudenberg

Masanori Ohya 
This page intentionally left blank 


\title{
Five years of QBIC
}

\author{
Masanori Ohya \\ Department of Information Sciences, \\ Tokyo University of Science, Japan
}

\section{Aims of QBIC}

The quantum bio-informatics center (QBIC) was founded in 2006 towards new stage making interdisciplinary bridges in philosophy, mathematics, physics, information and life sciences. Our research center (QBIC) tries to find a new paradigm for information science and life science on the basis of quantum mathematics. Several researchers more than 100 on mathematics, physics, information theory and biology who are interested in mathematical study worked together in QBIC during this five years from 2006 to 2010.

To solve the mystery of life is one of the most interesting problems in 21 century. After discovery of DNA, people believes that one key to read the riddle will be hidden in the process how information of life is stored and its change and transmission are made. Concerning the information transmission (communication), quantum information opened a new door and is expected to understand new aspects of existence in-itself.

More concretely, the immensely long DNA, sequence of four bases in the genome, contains information on life, and decoding or changing this sequence is involved in the expression and control of life. In quantum information, meanwhile, we produce various "information" by sequences of two quantum states, and think of ways of processing, communicating and controlling them. It is thought that the problems we can process in time " $\mathrm{T}$ " using a conventional computer can be processed in time nearly "log $\mathrm{T}$ " using a quantum computer. However, the transmission and processing of information in the living body might be much faster than those of quantum computer and communication.

Seen from this very basic viewpoint, developing the mathematical principles that have been found in quantum information should be useful in constructing mathematical principles for life sciences, which have not been established yet. The mechanism of processing information in life is also expected to be useful for the further growth of quantum information. 


\section{Research project}

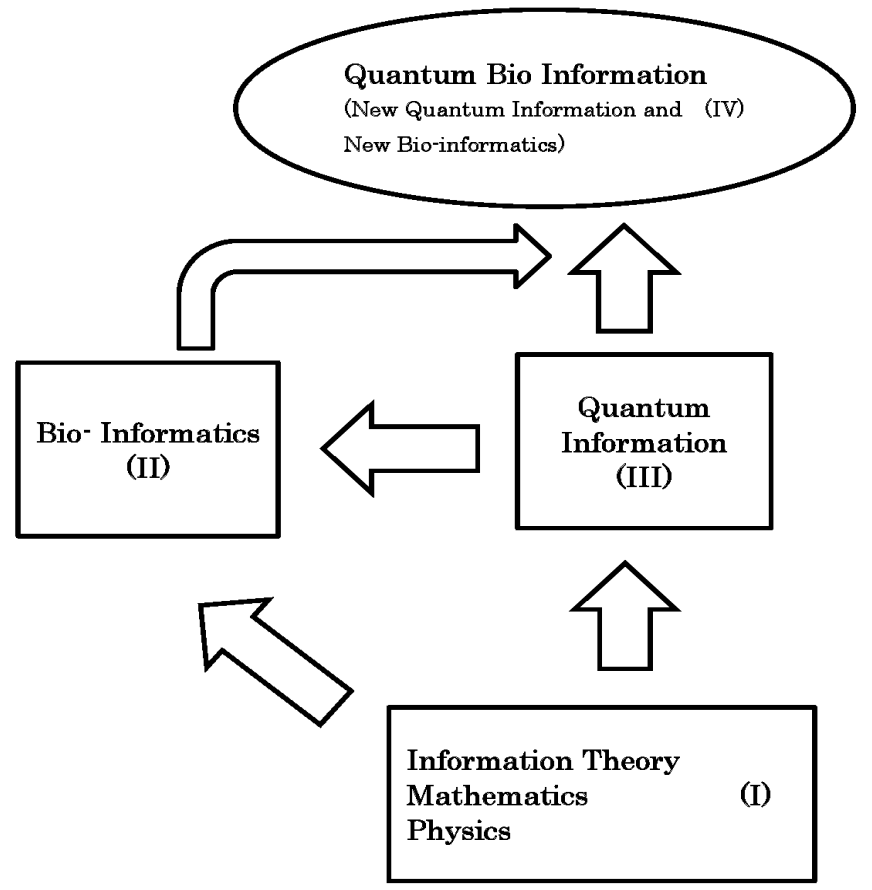

Figure 1. QBIC Research Project

The way of our research is

(1) to return to the starting point of bio-informatics and quantum information, fields that and to solve these fundamental problems, and

(2) to seriously attempt mutual interaction between the two, with a view to enumerating and solving the many fundamental problems they entail.

In our view, there is no similar research center in the world to return to the basics of bio-information and quantum information and to focus on the correlation between the two with a view to new development of each.

Our way with targets and goal are described in the figure below:

We had more than 200 papers published in this five years. Most of them have first published in the five proceedings of International Conference held in Tokyo University of Science. 
I will here review basic results of some achievements in this five years of QBIC.

\section{Solving the mystery of life}

Solving the mystery of life requires several stages (1) Metaphysical, (2) Biological \& Physical, (3) Mathematical. The works of the stages (1) and (2) have been done for a long time even in the "new" life science, that is, many philosophical considerations and various experiments have been done, and several (tentative) theories have been made. However it is also true we had not a basic mathematical rule (theory) in the life science so that many researchers could accept it as quantum mechanics.

In order to make such a theory, we have to try to develop fundamentals in various fields (mathematics, physics, information theory) with intention to the goal, i.e., finding the first principle understanding the life itself.

Biological systems are open systems. Biological systems are multicomponent and context dependent. Biological subsystems in a biological system are locally interacting each other. Therefore the state of the biological systems depends on its surrounding and the eve of itself. These observations entail that biological systems are adaptive. We have to find a mathematical rule to describe all of those.

However, in order to make our dream realize, we have to develop each field such as mathematics, quantum physics, information, structural biology and bio-informatics so that we can use the fructification to achieve new paradigm as discussed above.

\section{Some works appeared in conference of QBIC}

I itemize some works appeared in the conferences of QBIC during this five years. It is beyond the introduction and my ability to review all works appeared in the five years conferences, so that I only mention some mathematical trials somehow related to life sciences. The fruitful results of various works can been seen in the series of the five proceedings of QBIC conferences.

\subsection{Examples of researches in $Q B I C$}

\subsubsection{Concerning $<I>$ of the Figure above}

- White noise, stochastic analysis and some applications to DDS (Hida, Streit, SiSi, Accardi, Volovich, Smolyanov, Fichtner, In- 
oue, Iriyama, Hara, Ohya); Further developments of Hida calculas and its various applications have been considered, e.g., analysis of drug delivery systems.

- Mathematical physics and noncommutative analysis (Araki, Accardi, Arai, Belavkin, Jamiolkowski, Ojima, Petz, Hiai) have been developed so that we van apply to life sciences, for examples, quantum tomograhy, micro-macro duality.

- Statistics with symmetry (Tomizawa, Miyamoto, Tahata), which enables to analyze several fuctions of human body.

- Supercoductor $\Longrightarrow$ KS model and its development (Kamimura, Sakata, Ushio), which will be related to a realization of qubit.

- Fundamental problems in quantum physics - Bell's inequality, adaptive dynamics, quantum like systems, micro-macro duality, nonequilibrium dynamics (Accardi, Asano, Khrennikov, Volovich, Ojima, Suzuki, Oryu, Ohya)

- Ulitimate secure and fast crypto-algorithm can be found (Acradi, Regoli, Iriyama, Ohya).

\subsection{Concerning $<I I>$}

- Protein folding and simulation with brownian molecular dynamics (Yamato, Ando, Takeda, Im).

- Code structure of genes and works of cis-elements (Miyazaki, Sato, Khrennikov, Regoli, Wanke).

- Signal network of envitonmental sensing and adaptation in plants (Kuchitsu).

- Study of biosystems by information measures (R. Belavkin, A. Accardi, Im, Sato, Hara, Ohya), e.g., the most accurate alignment method is founded in QBIC.

\subsection{Concerning $<I I I>$}

- Quantum teleportation (Fichtner, Freudenberg, Kosakowski, Asano, Tanaka, Ohya), in which new mathematics and its realization are discussed, where the teleportation process can be linear and can use all entangled states.

- Quantum entanglement (Belavkin, Jamiolkowski, Accardi, Matsuoka, Kosakowski, Chruscinski, Majewski, Michalski, Hirota, Ohya), e.g., (1) we can treat even infinite systems; (2) we can treat all correlations including claasical one; (3) . 
- Quantum algorithm solving the NPC problem (Volovich, Accardi, Iriyama, Ohya) and its development; e.g., Our SAT alogithm can be used to solve factoring problem of Shor.

- Realization of qubit (Takayanagi, Morinaga).

- Quantum entropy and some applications (Accardi, Belavkin, Petz, Hiai, Araki, Iriyama, Matsuoka, Kosakowski,Watanabe, Suzuki, Ohya), e.g., Entropy production in linear respose dynamics, entropy production in photosythesis.

\subsection{Concerning $\langle I V\rangle$}

- Generalized Turing machine (Volovich, Iriyama, Ohya) is proposed.

- New description of chaos(Kosakowski, Togawa, Volovich, Inoue, Ohya $) \Longrightarrow$ Adaptive dynamics $\Longrightarrow$ Application of chaos dynamics to the classification of HIV-1 and Influenza A viruses (Sato, Tanabe, Hara)

- Non-Kolmogorov probability and its applications; Adaptive dynamics and lifting are applied to find new probability law (Khrennikov, Accardi, Asano, Basieva, Tanaka, Ohya, Yamato)

- Mathematical model explaining the fuctions of brain are proposed in Fock space (K.-H. Fichtner, L. Fichtner, Freudenberg, Inoue, Ohya).

- Quantum tomography and sufficiency (Jamiolkowski, Petz, Matsuoka, Watanabe, Ohya)

- Quantum algorithm solving the protein folding is studied (Goto, Iriyama, Yamato, Ohya)

- New alignment (MTRAP) of amino acids was proposed in terms of entanglement (Sato, Hara, Ohya)

- Alignment by means of quantum algorithm was made (Iriyama, Sato,Ohya)

- Game theory in non-Kolmogorovian probanility theory has been proposed (Khrennikov, Basieva, Asano, Tanaka, Ohya)

Remark New science must be based on new philosophy. Science (theory) without philosophy is fragile. The 21 century is the era not for new technology but for new philosophy crossing our existence.

Finally, I like to ask all of you interested in the QBIC conference: In order to understand the mystery of life and various existence, you dare to have will and intention to use all materials you obtained in several different fields. 


\section{References}

1. Accardi, L., Freudenberg, W., Ohya, M., Quantum Bio-Informatics (Quantum Probability and White Noise Analysis, Vol. 21), World Scientific, 2008

2. Accardi, L., Freudenberg, W., Ohya, M., Quantum Bio-Informatics II (Quantum Probability and White Noise Analysis, Vol. 24), World Scientific, , 2009

3. Accardi, L., Freudenberg, W., Ohya, M., Quantum Bio-Informatics III (Quantum Probability and White Noise Analysis, Vol. 26), World Scientific, 2010

4. Accardi, L., Freudenberg, W., Ohya, M., Quantum Bio-Informatics IV (Quantum Probability and White Noise Analysis, Vol. 28), World Scientific, 2011

5. Accardi, L., Freudenberg, W., Ohya, M., Quantum Bio-Informatics V (Quantum Probability and White Noise Analysis), World Scientific, this volume

6. Ohya, M., Volovich, I., Mathematical Foundations of Quantum Information and Computation and Its Applications to Nano- and Bio-systems, Springer, 2011 


\section{CONTENTS}

Preface $\quad$ V

Five Years of QBIC vii

Complexity Considerations Quantum Computation 1 Luigi Accardi

Quantum Markov Chains and Ising Model on Cayley Tree

Luigi Accardi, Farrukh Mukhamedov and Mansoor Saburov

Mathematical Aspects of Conserved Quantities in a General

Class of Quantum Systems

Asao Arai

Oscillations and Rolling for Duffing's Equation

Irina Ya. Aref'eva, Evgeny V. Piskovskiy and Igor V. Volovich

General Formalism of Decision Making Based on Theory of Open

Quantum Systems

Masanori Asano, Masanori Ohya, Irina Basieva and

Andrei Khrennikov

Quantum-Like Representation of Non-Bayesian Inference

Masanori Asano, Masanori Ohya, Irina Basieva, Andrei Khrennikov and Yoshiharu Tanaka

A Mathematical Treatment of Joint and Conditional Probability

Masanori Asano, Masanori Ohya, Yoshiharu Tanaka, Ichiro Yamato, Irina Besieva and Andrei Khrennikov

Entangled States Preparation in Clusters of Three Resonantly 
Minimum of Information Distance Criterion for Optimal Control of 95 Mutation Rate in Evolutionary Systems Roman V. Belavkin

On Non-Markovian Quantum Evolution

Dariusz Chruściński and Andrzej Kossakowski

High Density Limit of the Distribution of the Outcome of

EEG-Measurements

Karl-Heinz Fichtner, Lars Fichtner, Wolfgang Freudenberg and Masanori Ohya

Internal Noise of EEG-Measurements and Certain Boson Systems

Karl-Heinz Fichtner, Lars Fichtner, Kei Inoue and Masanori Ohya

Skew Information and Uncertainty Relation

Shigeru Furuichi and Kenjiro Yanagi

Multiple-Photon Absorption Attack on Entanglement-Based

Quantum Key Distribution Protocols

Guillaume Adenier, Noboru Watanabe, Irina Basieva and

Andrei Khrennikov

Protein Sequence Alignment Taking the Structure of Peptide Bond

Toshihide Hara, Keiko Sato and Masanori Ohya

Space - Time - Noise (Raum - Zeit - Rauschen)

Takeyuki Hida

Quantum Algorithm for Protein Folding and Its Computational Complexity

Satoshi Iriyama, Masanori Ohya and Ichiro Yamato

On Effective Procedures in Analyzing of Quantum Operations and Processes

Andrzej Jamiotkowski

On Numerical Ranges of Operators

Jacek Jurkowski 
Partial Roc Reveals Superiority of Mutual Rank of Pearson's

Correlation Coefficient as a Coexpression Measure to Elucidate

Functional Association of Genes

Takeshi Obayashi and Kengo Kinoshita

QFT and Hadronic World as Dynamical Bases of Natural History

237 Izumi Ojima

Long-Range Property in Time-Dependent Interaction with 253

Three-Body Structure and New Aspect

Shinsho Oryu

Kinetic Isotope Effect on Transport Mediated by

271

CLC-Type $\mathrm{H}^{+} / \mathrm{Cl}^{-}$Exchangers

Alessandra Picollo, Mattia Malvezzi and Alessio Accardi

On Positive Maps; Finite Dimensional Case

281

Wtadystaw A. Majewski

A New Noise Depending on a Space Parameter and Its Application

Si Si and Win Win Htay

Schrödinger Type Semigroups via Feynman Formulae and All That

301 Oleg G. Smolyanov

Entropy Production and Non-equilibrium Steady States Masuo Suzuki

Test and Measure on Difference of Asymmetry Between Several 327 Square Tables and Application to Medical Data

Kouji Tahata, Kouji Yamamoto, Nobuko Miyamoto and Sadao Tomizawa

Functional Mechanics and Kinetic Equations

Anton S. Trushechkin

Implications of DNA-Nanostructures by Hoogsteen-Dinucleotides on Transcription Factor Binding

Dierk Wanke, Luise H. Brand, Nina M. Fischer,

Florian Peschke, Joachim Kilian and Kenneth W. Berendzen 
On Treatment of Gaussian Communication Process by Quantum Entropies

Noboru Watanabe

Importance of Excluded Volume and Hydrodynamic Interactions on 375 Macromolecular Diffusion in vivo

Tadashi Ando and Jeffrey Skolnick

Self-Repelling Fractional Brownian Motion - A Generalized 389 Edwards Model for Chain Polymers

Jinky Bornales, Maria João Oliveira and Ludwig Streit

Signaling Networks Involving Reactive Oxygen Species and $\mathrm{Ca}^{2+}$ 403 in Plants

Kazuyuki Kuchitsu

A Novel Measure for Finding Disease-Specific Genes from the 409 Biomedical Literature

Yeondae Kwon, Hideaki Sugawara, Shogo Shimizu and Satoru Miyazaki

Three-Tangle and Three- $\pi$ for a Class of Tripartite Mixed States 425 Teng Ma and Shao-Ming Fei

Energy Flow and Information Flow in Superconducting Qubit Measurement Process

Hayato Nakano

How Can Steganography be an Interpretation of the Redundancy 447 in pre-mRNA Ribbon?

Massimo Regoli

Counter-factual Phenomenon in Quantum Mechanics Yutaka Shikano

From Structure and Function of Proteins Toward in Silico Biology 473 Ichiro Yamato 